\title{
Comparison of spread of subarachnoid sensory block and incidence of hypotension in early and late second trimester of pregnancy
}

\author{
Mi Hyeon Lee ${ }^{1}$, Hee Jeong Son ${ }^{2}$, Sang Hoon Lee ${ }^{1}$, Jeong-Hyun Lee ${ }^{1}$, Mi Hwa Chung ${ }^{1}$, \\ Young Ryong Choi ${ }^{1}$, and Eun Mi Choi ${ }^{1}$
}

Department of Anesthesiology and Pain Medicine, ${ }^{1}$ Kangnam Sacred Heart Hospital, Hallym University College of Medicine, Seoul, ${ }^{2}$ Kangwon National University Medical School, Chuncheon, Korea

Background: Obstetric or non-obstetric surgery can be performed in pregnant women during the second trimester. We evaluated maximal sensory block level (MSBL), and other sensory block characteristics after spinal anesthesia in early and late second trimester pregnant women.

Methods: Forty-four pregnant women scheduled for cervical cerclage under spinal anesthesia in the early second trimester (Group E) or in the late second trimester (Group L) were enrolled in this study. Spinal anesthesia was performed at the $\mathrm{L} 3 / 4$ and $7 \mathrm{mg}$ of $0.5 \%$ hyperbaric bupivacaine was injected into the subarachnoid space. Hemodynamic variables, incidence of nausea/vomiting, ephedrine dose, and sensory block were recorded every 2.5 min during the first 15 min and $20 \mathrm{~min}$ after the injection. The MSBL, the time at which MSBL was achieved, and the time to the two-segment regression of the sensory level were also recorded.

Results: The maximum number of segments blocked was significantly greater in the Group L than in the Group E. The incidence of nausea/vomiting and hypotension, and dose of administrated ephedrine were significantly higher in the Group L than in the Group E. The mean arterial pressure during the $15 \mathrm{~min}$ after subarachnoid injection was significantly lower compared to the baseline value in the Group L.

Conclusions: The MSBL of spinal anesthesia with hyperbaric bupivacaine $7 \mathrm{mg}$ were T9 in the early and T5 in the late second trimester groups. Pregnant women in the late second trimester exhibited increased incidence of hypotension and need for ephedrine than women in the early second trimester. (Korean J Anesthesiol 2013; 65: 322-326)

Key Words: Bupivacaine, Pregnancy, Second trimester.

\footnotetext{
Received: March 20, 2013. Revised: April 11, 2013. Accepted: April 24, 2013.

Corresponding author: Eun Mi Choi, M.D., Department of Anesthesiology and Pain Medicine, Kangnam Sacred Heart Hospital, Hallym University College of Medicine, 948-1, Daerim 1-dong, Yeongdeungpo-gu, Seoul 120-950, Korea. Tel: 82-2-829-5230, Fax: 82-2-2227-7897, E-mail: emchoi96@hallym.or.kr

(c) This is an open-access article distributed under the terms of the Creative Commons Attribution Non-Commercial License (http:// creativecommons.org/licenses/by-nc/3.0/), which permits unrestricted non-commercial use, distribution, and reproduction in any medium, provided the original work is properly cited.
} 


\section{Introduction}

Spinal anesthesia is a preferred anesthetic technique in pregnant women in their second trimester due to minimal fetal drug exposure and reduced potential teratogenicity. In late stage of pregnant women, the volume of the lumbosacral subarachnoid space is reduced due to engorgement of epidural blood vessels resulting from compression of the inferior vena cava by the gravid uterus $[1,2]$. The enhanced spread of spinal anesthesia, comparable in magnitude with that observed in the term group, occurred in the second trimester group [1].

In a previous study, pregnant women in their second trimester exhibited a higher level of subarachnoid sensory block than that of non-pregnant women within 30 minutes of hyperbaric bupivacaine injection [3]. The maximum number of dermatomes blocked was greater by three dermatomes in the second trimester group compared to the non-pregnant group [3].

In a majority of the previous studies that evaluated the level of sensory block resulting from spinal anesthesia administered to pregnant women, grouping was done according to trimesters, a period of 13 weeks. Such groupings fell short of identifying the level of sensory block in the early and late periods of the second trimester. Moreover, there were a few studies on the spread of the subarachnoid sensory block in early and late second trimester pregnant women.

Therefore, we evaluated the maximal sensory block level (MSBL) in pregnant women during early and late second trimester undergoing spinal anesthesia with $7 \mathrm{mg}$ of hyperbaric bupivacaine for cervical cerclage. In addition, we compared side effects such as hypotension and the requirement for ephedrine.

\section{Materials and Methods}

This study was approved by the Institutional Review Board of our hospital and registered at http://cris.nih.go.kr. Written informed consent was obtained from all the subjects and data collection was carried out from November 2011 to March 2012. Forty-four pregnant women scheduled for cervical cerclage under spinal anesthesia in the early second trimester (Group E) or in the late second trimester (Group L) were enrolled in this study. All the patients were American Society of Anesthesiologists physical status I or II. Patients with contraindications to spinal anesthesia, including coagulopathy, local skin infection, and uncorrected hypovolemia, body weight $(<50 \mathrm{~kg}$, or $>100$ $\mathrm{kg}$ ), multiple pregnancy and patients who could not assume the lateral position for spinal anesthesia due to risk of amnionic membrane rupture were excluded from our study.

None of the patients received premedication. On arrival in the operating room, standard monitoring devices (Patient monitor M1205A, Philips, USA or Micro O2, Siemens, Germany), including an electrocardiogram, pulse oximetry and a noninvasive blood pressure cuff, were applied to the patients. Baseline systolic blood pressure (SBP), diastolic blood pressure (DBP), mean arterial pressure (MAP), and heart rate (HR) were recorded. Before spinal anesthesia, all patients intravenously received $8-10 \mathrm{ml} / \mathrm{kg}$ of crystalloid solution. Patients were placed on their right side and the puncture site was disinfected. Local anesthetic was infiltrated into the subcutaneous tissue or muscle at the L3/4 interspinous space. A 25-gauge Whitacre spinal needle was inserted using the midline approach. When a free flow of cerebrospinal fluid was confirmed, $7 \mathrm{mg}$ of $0.5 \%$ hyperbaric bupivacaine (Marcaine ${ }^{\circledR}$, Astra Zeneca, Sweden) was injected into the subarachnoid space at $0.1 \mathrm{ml} / \mathrm{sec}$. After performing spinal anesthesia, patients were placed in the supine position with a wedge under the right hip for five minutes and then in the lithotomy position for surgery. Since then cervical cerclage was started. In Group L, amniocentesis was performed add to cerclage for decompression of intrauterine pressure. The endpoint of the study was reached when the sensory block was below T10 in the recovery room, and the patients were transported to their room. The spinal anesthesia was performed by a second year resident.

We recorded the SBP, DBP, MAP, HR, incidence of nausea/ vomiting, ephedrine dose, and sensory block every $2.5 \mathrm{~min}$ during the first $15 \mathrm{~min}$ and $20 \mathrm{~min}$ after the injection. The sensory block level was assessed at the left midclavicular line using loss of light touch sensation with a alcohol swab. The MSBL, the time at which MSBL was achieved, and the time to the two-segment regression (TSR) of the sensory level were also recorded. Each dermatomal level was scored in sequence starting at L3 $=1$ and T6 = 10 for statistical analyses. An intervention blood pressure was calculated as $80 \%$ of the baseline blood pressure. If SBP fell below an intervention blood pressure, or $90 \mathrm{mmHg}$, or if the patient complained of nausea and vomiting, ephedrine 4 mg was injected intravenously. When the blood pressure drop continued, the same dose was repeatedly injected. Oxygen was supplied at the rate of $5 \mathrm{~L} / \mathrm{min}$ through a facemask during the surgery.

\section{Statistical analysis}

The statistical analysis was performed with SAS (version 9.2, SAS Inc., Cary, NC, USA). The data was expressed as the mean \pm $\mathrm{SD}$, median (range), or number of patients. The sample size was based on a previous study [3] which had a three-dermatome difference in maximal block height with an alpha error of 0.05 and a power of $90 \%$. Twenty-two patients per group were necessary to demonstrate statistical significance. To compare variables between the two groups, the Kolmogorov-Smirnov test was used to identify the variables with a normal distribution. Variables with a normal distribution were compared by independent t-tests 
and those without a normal distribution were compared by the Mann-Whitney $U$-test. The number of patients was compared between the groups using the chi-square test. Value comparisons for each measurement were also conducted using repeated measures of ANOVA. All significant results were also analyzed with Tukey's post hoc test to determine whether time points produced values significantly different from baseline values. A P value less than 0.05 was considered statistically significant.

Table 1. Patients' Demographic Data in the Early (Group E) and Late (Group L) Second Trimester of Pregnant

\begin{tabular}{lccr}
\hline & $\begin{array}{c}\text { Group E } \\
(\mathrm{n}=22)\end{array}$ & $\begin{array}{c}\text { Group L } \\
(\mathrm{n}=22)\end{array}$ & P value \\
\hline Age $(\mathrm{yr})$ & $33.0 \pm 2.8$ & $32.3 \pm 3.2$ & 0.3961 \\
Height $(\mathrm{cm})$ & $162.5 \pm 4.1$ & $161.4 \pm 6.4$ & 0.5044 \\
Weight $(\mathrm{kg})$ & $62.2 \pm 9.8$ & $63.0 \pm 10.1$ & 0.8098 \\
Gestational age (week) & $13.3 \pm 0.6$ & $22.5 \pm 2.0$ & $<0.0001$ \\
Weight of fetus $(\mathrm{g})$ & $89.0 \pm 7.6$ & $616.6 \pm 196.0$ & $<0.0001$ \\
\hline
\end{tabular}

Values are presented as the mean \pm SD.

Table 2. Sensory Block Characteristics in the Early (Group E) and Late (Group L) Second Trimester of Pregnant

\begin{tabular}{lccr}
\hline & $\begin{array}{c}\text { Group E } \\
(\mathrm{n}=22)\end{array}$ & $\begin{array}{c}\text { Group L } \\
(\mathrm{n}=22)\end{array}$ & P value \\
\hline Nausea/Vomiting (n) & 0 & 5 & 0.0485 \\
Incidence of hypotension (n) & 0 & 7 & 0.0089 \\
Dose of ephedrine (mg) & 0 & $4.7 \pm 8.5$ & 0.0100 \\
MSBL (dermatome) & $7.3 \pm 2.1$ & $11.0 \pm 2.7$ & $<0.0001$ \\
Time to MSBL (min) & $11.7 \pm 4.3$ & $10.8 \pm 2.9$ & 0.4186 \\
Time to TSR (min) & $58.9 \pm 14.8$ & $55.0 \pm 13.8$ & 0.3755 \\
\hline
\end{tabular}

Values are presented as the mean \pm SD or number of patients. Dermatome $1=\mathrm{L} 3 ; 4=\mathrm{T} 12 ; 8=\mathrm{T} 8 ; 12=\mathrm{T} 4$ of sensory block level. MSBL: maximal sensory block level, TSR: two-segment regression.

A

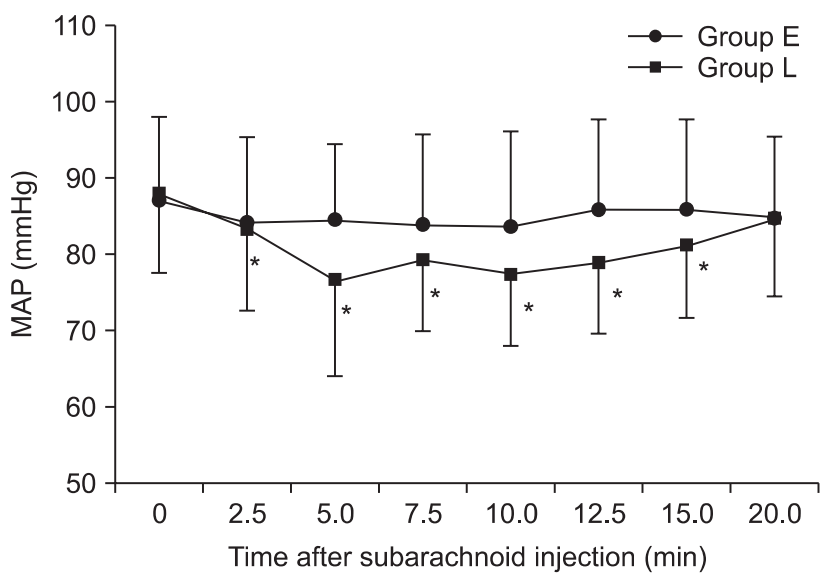

\section{Results}

Forty-four pregnant women were enrolled in this study and none were excluded or failed to inadequate anesthesia.

The demographic data of the patients is presented in Table 1. The mean gestational age was $13.3 \pm 0.6$ in Group E vs $22.5 \pm$ 2 weeks in Group $\mathrm{L}$ and the fetal weight measured by ultrasonography was $89 \pm 7.6$ vs $616.6 \pm 196 \mathrm{~g}$ in the Group E and L, respectively. The other characteristics of the patients were not significantly different between the groups.

The number of dermatomes blocked at each time point is shown in Fig. 1. The number of dermatomes blocked was higher in the Group L 2.5 min after the subarachnoid injection. The maximum number of segments blocked was significantly greater

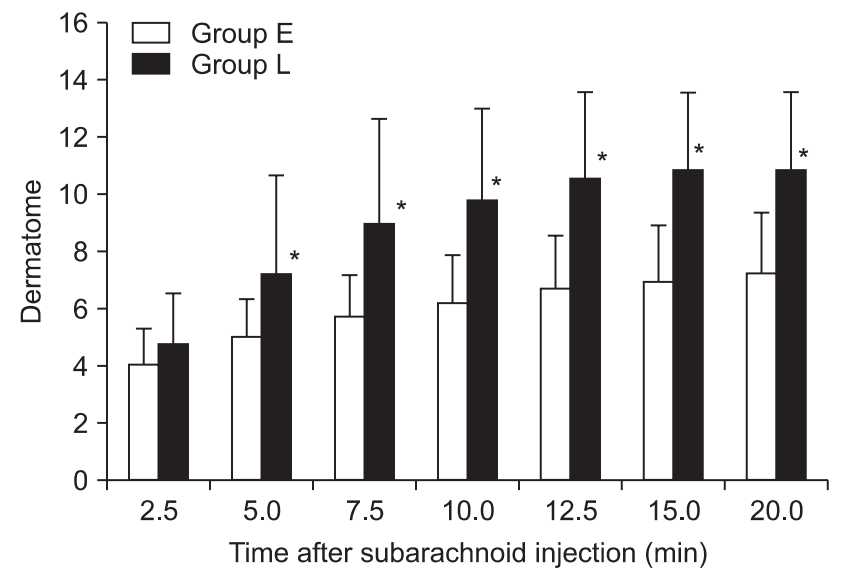

Fig. 1. Number of dermatomes blocked in the early (Group E) and late (Group L) second trimester of pregnant at 2.5, 5, 7.5, 10, 12.5, 15 and 20 minutes after subarachnoid injection of $7 \mathrm{mg}$ of $0.5 \%$ bupivacaine. ${ }^{*} \mathrm{P}<$ 0.05 . Data represent mean $\pm \mathrm{SD}$. Dermatome $1=\mathrm{L} 3 ; 4=\mathrm{T} 12 ; 8=\mathrm{T} 8 ; 12$ $=\mathrm{T} 4$ of sensory block level.

B

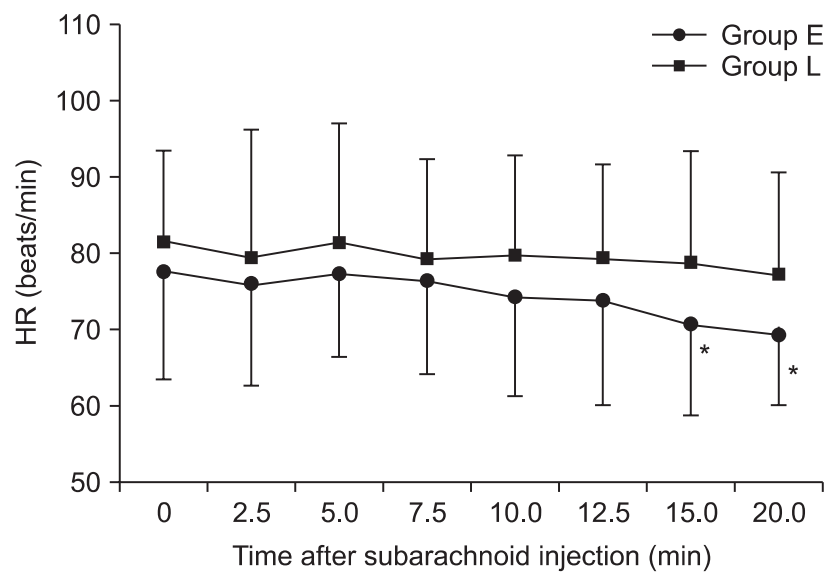

Fig. 2. Changes in mean arterial pressure (MAP, A) and heart rate (HR, B) after subarachnoid injection of $7 \mathrm{mg}$ of $0.5 \%$ bupivacaine in the early (Group E) and late (Group L) second trimester of pregnant. Data represent mean $\pm \mathrm{SD}$. $* \mathrm{P}<0.05$ compared with baseline value in each group. 
in the Group L than in the Group E $(11.0 \pm 2.7$ vs $7.3 \pm 2.1, \mathrm{P}<$ $0.0001)$.

The characteristics of the sensory blocks are presented in Table 2. The incidence of nausea/vomiting and hypotension, and dose of administrated ephedrine were significantly higher in the Group L than in the Group E. However, the time at which MSBL was achieved, and the time to the TSR of the sensory level were not significantly different between the two groups.

Fig. 2 shows the changes in MAP and HR after the subarachnoid injection. The MAP and HR were not significantly different during the study period between the two groups. However, the MAP during the first $15 \mathrm{~min}$ after subarachnoid injection was significantly lower compared to the baseline value of the Group L.

\section{Discussion}

In our study, the maximal sensory block levels of spinal anesthesia with hyperbaric bupivacaine $7 \mathrm{mg}$ were $\mathrm{T} 9$ in the early and $\mathrm{T} 5$ in the late second trimester groups. Furthermore, pregnant women in the late second trimester exhibited a higher incidence of hypotension and required more frequently ephedrine than that of the early second trimester.

The mechanism for the enhanced spread of spinal anesthesia in pregnant women has not been clearly elucidated. A mechanical factor that could be one possible explanation is the decreased CSF volume at lumbosacral area caused by the distension of the extradural veins due to obstruction of the inferior vena cava by the gravid uterus [4,5] and changes in the curvature of the spinal column [6]. Epidural venous distension develops in the first trimester and remains throughout the third trimester [7]. Lee and colleagues [3] investigated the level of sensory block after spinal anesthesia in pregnant women in their second trimester (15.7 weeks mean gestational age). In their study, the maximal block level with hyperbaric bupivacaine $7 \mathrm{mg}$ was $\mathrm{T} 7$ in the second trimester and it was greater by three dermatomes than in the nonpregnant group. When considering the mean gestational age, this result was similar to our study in that patients in the early and late second trimester group had maximal sensory block levels of T9 and T5, respectively. Although compression of the vena cava by the enlarged uterus mechanically did not occur in the early second trimester, venous distension secondary to the increased blood volume associated with pregnancy did occur [8]. In our study, fetal weight measured by ultrasonography was 89.0 \pm 7.6 vs $616.6 \pm 196.0$ gm in the Group E and L, respectively. In the late second trimester group $(22.5 \pm 2.0$ weeks mean gestational age), the increased size of the fetus and the enlarged uterus might compress the vena cava similar to the third trimester.

Another mechanical factor that could explain our findings is the change in the curvature of the spinal column. Lumbar lordosis exaggerated by the enlarged uterus at term pregnancy is thought to influence the spread of spinal anesthesia [9]. However, the effect from the mechanical factor on the difference in the spread of spinal anesthesia in the early and late second trimester does not seem to provide a sufficient explanation by itself.

As a non-mechanical factor, plasma and CSF progesterone has been suggested as a reason for the enhanced spread of spinal anesthesia by altering the susceptibility of the nerves to local anesthetics [10-12]. Hirabayashi et al. [10] measured the progesterone concentration of the CSF in non-pregnant, first, second, third trimester, and term pregnant women. They observed that the progesterone concentration of the CSF in third trimester and term pregnant women was higher than in non-pregnant, first, and second trimester pregnant women. However, the maximal cephalad spread of spinal analgesia was higher in second, third trimester, and term pregnant women. Their study suggests that not only a minimum level of progesterone in the CSF but also a certain period of exposure may be necessary for the enhanced spread of spinal anesthesia. Bader et al. [13] investigated the effect of progesterone on neural blocks although the cellular mechanism of progesterone is unclear, and found that a development period for increased sensitivity of the nerves to local anesthetics was needed. A certain period of exposure to elevated levels of progesterone resulting in increased sensitivity to local anesthetics is thought to be probable explanation for a more enhanced spread of spinal anesthesia in late second trimester pregnancy than in early second trimester pregnancy.

Hemodynamic changes including blood pressure and HR are observed at the early and middle stages of pregnancy. SBP decreases by $6-8 \%$ at the middle stages of pregnancy and HR increases at the second trimesters by $20 \%[14,15]$. In our study, although we could not compare the hemodynamic changes with non-pregnant women, we were able to investigate the cardiovascular response to spinal anesthesia in the early and late second trimester. The incidence of nausea/vomiting and hypotension, and dose of administrated ephedrine were significantly higher in the Group L than in the Group E. Seven out of twenty-two patients in the late second trimester group had hypotension occur after spinal anesthesia with hyperbaric bupivacaine $7 \mathrm{mg}$. Our results possibly suggest that cardiovascular responses to spinal anesthesia are different between early and late second trimester pregnancy. The time at which MSBL was achieved, and the time to the TSR of the sensory level were faster in the Group L, but did not have any statistical difference.

This study differentiates itself from previous studies in that it divides the second trimester into early and late spans. Previous studies were mostly designed to regard the second trimester as a sole unit. This study revealed that the level of sensory block of spinal anesthesia differs within the second trimester according to the number of gestation weeks.

Our study has some limitations. First, after performing spinal 
anesthesia, patients were turned to the supine position for five minutes and then fixed in the lithotomy position during the operation. It is thought that the lithotomy position can limit the cephalad spread of local anesthetics because of the loss of the lumbosacral curvature. However, according to a study by Logan and Drummond [16], limiting hip flexion to $90^{\circ}$ does not alter the level of anesthetic sensory block. Second, our study was not blinded for the patients and observers because the amniocentesis was added to cervical cerclage in Group L for decompression.

In conclusions, the MSBL of spinal anesthesia with hyper- baric bupivacaine $7 \mathrm{mg}$ were T9 in the early and T5 in the late second trimester groups. Pregnant women in the late second trimester exhibited increased incidence of hypotension and need for ephedrine than women in the early second trimester.

\section{Acknowledgments}

We thank Hye Sun Lee, Biostatistician, Department of Research Affairs, Yonsei University College of Medicine, for statistical analysis.

\section{References}

1. Birnbach DJ, Brown IM. Anesthesia for obstetrics. In: Miller's Anesthesia. 6th ed. Edited by Miller RD: Philadelphia, Elsevier/Churchill Livingstone. 2005, p 2324.

2. Higuchi H, Hirata J, Adachi Y, Kazama T. Influence of lumbosacral cerebrospinal fluid density, velocity, and volume on extent and duration of plain bupivacaine spinal anesthesia. Anesthesiology 2004; 100: 106-14.

3. Lee GY, Kim CH, Chung RK, Han JI, Kim DY. Spread of subarachnoid sensory block with hyperbaric bupivacaine in second trimester of pregnancy. J Clin Anesth 2009; 21: 482-5.

4. Barclay DL, Renegar OJ, Nelson EW Jr. The influence of inferior vena cava compression on the level of spinal anesthesia. Am J Obstet Gynecol 1968; 101: 792-800.

5. Bromage PR. Continuous lumbar epidural analgesia for obstetrics. Can Med Assoc J 1961; 85: 1136-40.

6. Hirabayashi Y, Shimizu R, Fukuda H, Saitoh K, Furuse M. Anatomical configuration of the spinal column in the supine position. II. Comparison of pregnant and non-pregnant women. Br J Anaesth 1995; 75: 6-8.

7. Igarashi T, Hirabayashi Y, Shimizu R, Saitoh K, Fukuda H, Suzuki H. The fiberscoptic findings of the epidural space in pregnant women. Anesthesiology 2000; 92: 1631-6.

8. Marx G, Bassell G. Physiologic considerations of the mother. In: Obstetric Analgesia and Anesthesia. Edited by Marx G, Bassell G: New York, Elsevier/North-Holland. 1980, pp 21-54.

9. Greene NM. Distribution of local anesthetic solutions within the subarachnoid space. Anesth Analg 1985; 64: 715-30.

10. Hirabayashi Y, Shimizu R, Saitoh K, Fukuda H. Cerebrospinal fluid progesterone in pregnant women. Br J Anaesth 1995; 75: 683-7.

11. Fagraeus L, Urban BJ, Bromage PR. Spread of epidural analgesia in early pregnancy. Anesthesiology 1983; 58: 184-7.

12. Datta S, Hurley RJ, Naulty JS, Stern P, Lambert DH, Concepcion M, et al. Plasma and cerebrospinal fluid progesterone concentrations in pregnant and nonpregnant women. Anesth Analg 1986; 65: 950-4.

13. Bader AM, Datta S, Moller RA, Covino BG. Acute progesterone treatment has no effect on bupivacaine-induced conduction blockade in the isolated rabbit vagus nerve. Anesth Analg 1990; 71: 545-8.

14. Capeless EL, Clapp JF. Cardiovascular changes in early phase of pregnancy. Am J Obstet Gynecol 1989; 161: 1449-53.

15. Clapp JF 3rd, Seaward BL, Sleamaker RH, Hiser J. Maternal physiologic adaptations to early human pregnancy. Am J Obstet Gynecol 1988; 159: 1456-60.

16. Logan MR, Drummond GB. Spinal anesthesia and lumbar lordosis. Anesth Analg 1988; 67: 338-41. 